\title{
МЕДИКАМЕНТОЗНЫЙ РИНИТ
}

\section{ВОПРОСЫ И ОТВЕТЫ}

\begin{abstract}
Медикаментозный ринит (МР) развивается в результате длительного приема топических деконгестантов и различных лекарственных препаратов системного действия. В статье представлен обзор литературных данных, освещающих вопросы патогенеза, клиники, диагностики и лечения заболевания. Подчеркнуто, что в настоящее время интраназальные глюкокортикостероиды являются единственными препаратами, эффективность которых при МР доказана в рандомизированных контролируемых клинических исследованиях. Авторы приводят собственные наблюдения применения мометазона фуроата у 16 больных с МР в течение 4 недель при режиме дозирования 400 мкг/сут. 12 пациентов (75\%) смогли полностью отказаться от приема деконгестантов к концу второй недели лечения. В одном случае имел место рецидив заболевания. Топические стероиды позволяют облегчить пациенту период отказа от топических сосудосуживающих препаратов. Тем не менее больные должны быть предупреждены о возможности рецидива МР в случаях повторного бесконтрольного использования деконгестантов.
\end{abstract}

Ключевые слова: медикаментозный ринит, мометазона фуроат.

V.V. SHELENKOVA, MD, Prof., Yaroslavl State Medical University DRUG-INDUCED RHINITIS: QUESTIONS AND ANSWERS

Drug-induced rhinitis (DIR) develops as a result of long-term use of topical decongestants and different drugs of systemic action. The article presents a review of the literature covering the issues of pathogenesis, clinic, diagnostics and treatment of the disease. It is stressed that currently intranasal glucocorticosteroids are the only drugs effectiveness of which for drug-induced rhinitis is proved in randomized controlled clinical trials. The authors provide observations of the use of mometasone furoate in 16 patients with DIR for 4 weeks at a dosing of $400 \mathrm{mg} /$ day. 12 patients $(75 \%)$ were able to completely stop taking decongestants to the end of the second week of treatment. In one case there was recurrence of the disease.

Topical steroids help to alleviate the patient's period of withdrawal from topical vasoconstrictor preparations. However, patients should be warned about the possibility of recurrence of DIR in cases of repeated uncontrolled use of decongestants.

Keywords: drug-induced rhinitis, mometasone furoate.

T ермин «медикаментозный ринит» (МР) чаще всего используется для описания длительной заложенности носа у пациентов, которые неоднократно использовали назальные деконгестанты. Первым назальным сосудосуживающим препаратом в истории ринологии стал эфедрин. Он был синтезирован в 1887 г. из травы ma-huang и использовался в виде назальных ингаляций, спреев и капель для снятия отека в полости носа [1]. Лишь спустя чуть менее полувека, в 1931 г., N. Fox впервые описал побочные эффекты эфедрина, которые впоследствии получили название «синдром рикошета» [2]. Впервые эту терминологию применили S.M. Feinberg и S. Friedlaender в 1944 г., а в 1946 г. C.F. Lake предложил термин «медикаментозный ринит» [3, 4].

Несмотря на давнюю историю заболевания, концепция «рикошета» до сих пор ставится под сомнение, а диагностические критерии МР остаются весьма неопределенными, так как в большинстве случаев заложенность носа, являющаяся основным симптомом в постановке диагноза, относится к весьма субъективным клиническим признакам [5]. В связи с этим постараемся ответить хотя бы на некоторые вопросы, касающиеся МР.

Прежде всего, что такое МР? Согласно современным представлениям о данном заболевании медикаментозным называется ринит, индуцированный длительным приемом различных лекарственных препаратов, при этом необязательно деконгестантов. В настоящее время условно выделяют две разновидности этой болезни: ринит, обусловленный местным применением сосудосуживающих средств, и ринит, возникший вследствие регулярного использования различных лекарственных препаратов системного действия, в частности нейролептиков, гипотензивных средств, ингибиторов 5-фосфодиэстеразы, гормонов (эстрогенов), нестероидных противовоспалительных препаратов (аспирина).

Механизмы неблагоприятного воздействия системных препаратов на слизистую оболочку полости носа различны. Например, использующиеся для лечения артериальной гипертензии блокаторы а-адренорецепторов препятствуют прохождению сосудосуживающих импульсов через адренергические синапсы и вызывают расширение артериол и прекапилляров, т. е. по своей сути вызывают вазодилататорный эффект. Симпатолитики, основными представителями которых являются резерпин, гуанетидин и бретилия тозилат, снижают количество норадреналина, выделяющегося в окончаниях симпатических нервов, в результате чего происходит торможение передачи адренергического возбуждения. Эти препараты сейчас редко используются в практике. Сосудорасширяющий эффект ацетилсалициловой кислоты объясняется ингибированием циклооксигеназы, что обуславливает снижение уровня простагландинов в тканях. 
Патогенез развития МР в ответ на длительное применение топических деконгестантов до сих пор изучен недостаточно. Тем не менее существует несколько гипотез. Согласно одной из них МР является следствием длительной вазоконстрикции и ишемии, вызванной интенсивной стимуляцией a2-адренорецепторов слизистой оболочки полости носа назальными деконгестантами [6, 7]. Другая гипотеза заключается в эффекте обратной связи, т. е. нарушении нервной регуляции слизистой оболочки вследствие повторяющейся на протяжении длительного времени стимуляции а2-адренорецепторов, приводящей к уменьшению числа функциональных рецепторов и снижению их чувствительности. Адренорецепторы становятся невосприимчивыми к топическим деконгестантам, развивается так называемая тахифилаксия, в результате чего пациенту приходится увеличивать дозировку препарата для получения должного эффекта [8, 9]. В конечном итоге эти процессы приводят к вазодилатации и венозному застою в полости носа [5, 10, 11]. В большей степени это касается кавернозной ткани нижних носовых раковин. Кроме того, существуют исследования, подтверждающие неблагоприятное воздействие на слизистую оболочку полости носа консервантов, входящих в состав сосудосуживающих средств. Чаще всего в качестве консервантов используется бензалкония хлорид. Доказано, что нанесение этого вещества на слизистую оболочку в течение 30 дней приводит к появлению стойкого отека в полости носа у абсолютно здоровых людей [12]. В целом можно утверждать, что топические деконгестанты способствуют нарушению баланса вегетативной нервной системы на уровне полости носа, при этом преобладает парасимпатическая импульсация, что проявляется изменением тонуса сосудов и развитием отека слизистой оболочки $[13,14]$.

Насколько быстро развивается МР? Ответить на этот вопрос однозначно не представляется возможным ввиду многообразия данных, полученных в ходе различных исследований на добровольцах, причем с использований разных методов оценки. Чаще всего в этих работах в качестве топического деконгестанта использовался оксиметазолин, а в качестве методов оценки носового дыхания - риноманометрия (определение носового сопротивления воздушной струи, проходящей через полость носа при свободном дыхании) и риностереометрия (измерение толщины слизистой оболочки носа). Так, по данным S. Morris et al. (1997), значительное нарастание носового сопротивления в полости носа наблюдается уже после 3 дней применения оксиметазолина [15]. Исследования Р. Graf (1996) свидетельствуют о возможном нарастании отека в полости носа через 10 дней регулярного нанесения на слизистую оболочку топического деконгестанта [11]. В работе S. Vaidyanathan et al. (2010) эти сроки уже значительно больше и составляют в среднем 17 дней [10]. Тем не менее некоторые литературные данные позволяют усомниться в возможности такого быстротечного развития МР. Так, ряд авторов не наблюдали ухудшения носового дыхания у здоровых лиц на протяжении 4 недель терапии оксиметазолином $[16,17]$.
Каковы патоморфологические аспекты МР? Ответить на этот вопрос так же сложно, как и на предыдущие. Во-первых, серьезных клинических исследований по данному направлению не проводилось. Во-вторых, развитие МР в большинстве случаев обусловлено длительным применением топических деконгестантов у лиц, уже страдающих какой-либо патологией полости носа, например хроническим риносинуситом, деформацией носовой перегородки, аномалиями внутриносовых структур, аллергическим ринитом. Поэтому четко сформулировать специфичные признаки изменений слизистой оболочки полости носа при МР невозможно. Вероятнее всего, к этим признакам можно отнести метаплазию назального эпителия с нарушением функции реснитчатых клеток вплоть до полной потери ресничек, гиперплазию бокаловидных клеток и секреторных желез, гиперсекрецию слизи, повышенную васкуляризацию слизистой оболочки с последующим развитием фиброза [18-20].

\section{В челом можно утверждать, что топические деконгестанты способствуют нарушению баланса вегетативной нервной системы на уровне полости носа, при этом преобладает парасимпатическая импульсачия, что проявляется изменением тонуса сосудов и развитием отека слизистой оболочки}

Как проявляется МР? Ведущим клиническим симптомом заболевания является длительно персистирующая заложенность носа. Выраженность затруднения носового дыхания различна вплоть до полного его отсутствия, когда пациент дышит преимущественно ртом, при этом выражена гнусавость и нарушено обоняние. Выделения из носа, как правило, не характерны, так же как и зуд в полости носа и чиханье. Тем не менее постоянная заложенность носа приводит к значительному нарушению качества жизни пациента, становится причиной расстройства сна, снижения социальной активности и когнитивных функций.

Постановка диагноза МР, как правило, не вызывает особых трудностей и не требует проведения специальных лабораторных и инструментальных исследований. Основными диагностическими признаками болезни являются три критерия, которые были сформулированы J.S. Walker еще в 1952 г., но по-прежнему остаются актуальными: 1) анамнез, а именно история длительного применения лекарственных препаратов, прежде всего деконгестантов; 2) констатация факта постоянной назальной обструкции; 3) уменьшение отека слизистой оболочки полости носа при воздействии вазоконстрикторов [21]. Тем не менее необходимо учитывать, что назальные деконгестанты используются для улучшения носового дыхания при различных заболеваниях: остром рините на фоне вирусной инфекции, аллергическом рините, рините беременных, аденоидах, остром и хроническом риносинусите, полипах носа, назальной обструкции при деформации носовой перегородки и прочих состояниях [7]. Иначе говоря, МР всегда имеет определенную «почву» для своего развития, 
поэтому необходимо не только констатировать факт назальной обструкции, но и выяснить причину, которая привела к необходимости длительного приема деконгестантов, и по возможности ее устранить. С этой целью прибегают к эндоскопии полости носа, компьютерной томографии околоносовых пазух, бактериологическому анализу материала из полости носа, риноманометрии.

Какую терапию следует провести пациенту, страдающемy MP? Лечение МР представляет собой весьма сложную задачу, порой неразрешимую, т. к. требует соблюдения двух основных условий. Это - настойчивость врача и мотивация со стороны больного, причем последнее далеко не всегда возможно добиться у определенной категории пациентов. Поэтому начать необходимо с разъяснения пациенту исходов заболевания и возможных методов лечения.

\section{Единственными препаратами, эффективность которых при МР доказана не только в эксперимен- тальных, но и в клинических рандомизированных плацебо-контролируемых исследованиях, являются интраназальные глюкокортикостероиды}

Главное в лечении МР - отказ от топических сосудосуживающих препаратов. Считается, что внезапное прекращение приема деконгестантов может привести к усилению отека слизистой оболочки полости носа. Поэтому делать это необходимо постепенно, на фоне медикаментозной терапии. С этой целью предлагались пероральные антигистаминные препараты, седативные и снотворные средства, оральные глюкокортикостероиды, солевые назальные спреи, назальные капли с дексаметазоном, триамцинолоном, кромолином, инъекции глюкокортикостероидов в нижние носовые раковины. Однако эффективность этих методик и лекарственных средств никогда не была подтверждена в рандомизированных исследованиях [20].

Единственными препаратами, эффективность которых при МР доказана не только в экспериментальных, но и в клинических рандомизированных плацебо-контролируемых исследованиях, являются интраназальные глюкокортикостероиды (ИнГКС). Первая работа об успешном излечении МР топическими стероидами была опубликована в 1968 г., однако количество наблюдений авторов не превысило 4 пациентов [22]. Затем появились более серьезные публикации. В одной из них приведены результаты наблюдения за 10 пациентами с МР, которые в течение 6 недель были успешно пролечены интраназальным будесонидом с полным отказом от приема деконгестантов [11]. Позднее в рандомизированном двойном слепом контролируемом исследовании была доказана эффективность двухнедельного применения спрея флутиказона пропионата в сравнении с плацебо у 20 пациентов с МР. При этом в качестве критериев эффективности ИнГКС авторы использовали риностереометрию и акустическую ринометрию, которые считаются стандартизированными методами для объективной оценки носового дыхания [6, 23].

Интересные экспериментальные исследования были проведены в отношении другого ИнГКС - мометазона фуроата. Авторы индуцировали развитие МР у 24 морских свинок путем инсталляции им в нос оксиметазолина в течение 8 недель. Затем животные были разделены на три группы. В первой группе морским свинкам в течение 2 недель инсталлировали в нос мометазон, во второй группе 0,9\%-ный раствор хлорида натрия, третью группу животных не лечили. Проведенные в последующем гистологические исследования показали прогрессивное снижение отека слизистой оболочки полости носа у морских свинок под воздействием мометазона, что подтвердило целесообразность применения препарата в терапии МР [24].

Мометазона фуроат относится к синтетическим глюкокортикостероидам для местного применения. Основной механизм его действия состоит в торможении высвобождения медиаторов воспаления и нарушении каскада арахидоновой кислоты, за счет чего предупреждается краевое скопление нейтрофилов, уменьшается продукция лимфокинов и образование воспалительного экссудата, тормозится миграция макрофагов, что приводит к уменьшению процессов инфильтрации и грануляции. Преимущество мометазона фуроата перед другими препаратами группы ИнГКС состоит в пренебрежимо малой биодоступности $(<0,1 \%)$, что обеспечивает безопасность его применения даже в течение длительных курсов. Кроме того, серьезные лабораторные исследования, проведенные за рубежом, доказали отсутствие негативного влияния препарата на слизистую оболочку полости носа. В частности, в работах E. Minshall et al. показано, что длительное применение мометазона фуроата не вызывает формирования в слизистой оболочке деструктивных процессов. Напротив, препарат способствует восстановлению целостности эпителиального покрова полости носа, а также редукции клеточных инфильтратов [25]. Данный факт можно считать одним из основополагающих в определении целесообразности применения препарата в лечении МР, поскольку, как правило, требуется длительный, не менее месяца, прием топического стероида.

Из последних работ, посвященных успешному применению ИнГКС при МР, внимание заслуживает публикация японских исследователей [26]. Авторы использовали разные топические стероиды у 33 пациентов с МР. В результате 31 пациент отказался от деконгестантов в течение 4 недель. Большинство (61,3\%) отметило улучшение уже в течение первых 3 дней приема ИнГКС, через 1 неделю количество таких больных составило уже 80,6\%. В связи с этим считаем целесообразным представить собственные данные.

Под нашим наблюдением находилось 16 пациентов с MP: 11 мужчин и 5 женщин в возрасте от 20 до 56 лет. Из них 6 пациентов (37,5\%) регулярно принимали топические деконгестанты меньше 1 года, но не менее 5 месяцев. 10 (62,5\%) больных ежедневно использовали деконгестанты более года, причем 1 пациентка - больше 5 лет. В большинстве случаев причиной длительного приема сосудосуживающих средств был симптом неконтролируемой назальной обструкции после перенесенной вирусной инфекции (11 пациентов). Два пациента страдали персистирующим аллергическим ринитом. Еще у 2 в 
анамнезе имелись указания на хронический риносинусит без полипов. В 1 случае пациент не смог назвать причину злоупотребления деконгестантами, однако пользовался ими уже более 2 лет.

Всем пациентам произведена эндоскопия полости носа, выполнена компьютерная томография околоносовых пазух. При эндоскопическом исследовании во всех случаях имел место отек слизистой оболочки нижних носовых раковин. У 5 больных выявлена деформация носовой перегородки в виде гребня в заднем отделе полости носа либо на всем протяжении перегородки носа. В одном случае (женщина 24 лет) диагностирована гипертрофия глоточной миндалины II степени. Из клинически значимых находок на компьютерных томограммах обращали на себя внимание буллезные средние носовые раковины (3 случая), киста верхнечелюстных пазух (2).

С каждым пациентом проведена беседа о целесообразности отказа от сосудосуживающих препаратов и курения, а также проведения длительной топической глюкокортикостероидной терапии. С этой целью мы предпочли использовать мометазона фуроат курсом не менее 4 недель в суточной дозировке 400 мкг с режимом приема по 2 спрея в каждую половину носа утром и вечером. Пациентам рекомендовали первые 10 дней проводить ирригационную терапию полости носа за 15-20 минут до приема препарата. В течение первой недели терапии ИнГКС допускалось применение деконгестантов, но только в одну половину носа и лишь перед сном. Предполагалось, что пациенты должны полностью отказаться от деконгестантов на второй неделе лечения. При этом прием ИнГКС не прекращали вплоть до улучшения носо- вого дыхания настолько, что пациенту не требовались сосудосуживающие препараты, но не менее 4 недель.

В целом из 16 пациентов 12 (75\%) смогли полностью отказаться от деконгестантов. Из них у 9 значимое улучшение носового дыхания наступило к концу первой недели, т.е. на 5-7-й день приема мометазона фуроата, у 3 на второй неделе, через 10-12 дней от начала лечения. В дальнейшем 4 пациента были прооперированы по поводу деформаций внутриносовых структур, им выполнена септопластика с резекцией буллезных средних носовых раковин. Двум больным произведена микрогайморотомия, в 1 случае - аденотомия.

Один пациент самостоятельно прервал прием препарата на второй неделе лечения и выбыл из исследования. Дальнейшая судьба его не известна. В 3 случаях эффекта не получено. Эти пациенты направлены на хирургическую редукцию нижних носовых раковин. В дальнейшем один из них вновь стал принимать деконгестанты на фоне вирусной инфекции и обратился повторно через 1,5 года после лечения с теми же жалобами.

Таким образом, в настоящее время ИнГКС следует считать препаратами выбора для лечения МР. Благодаря противовоспалительному эффекту топические стероиды позволяют облегчить пациенту процесс отказа от приема топических сосудосуживающих препаратов. Тем не менее больные должны быть предупреждены о возможности рецидива МР в случаях повторного использования деконгестантов и информированы о том, что прием препаратов должен быть ограничен сроками, указанными в инструкции к лекарственному средству, по крайней мере не более 7-10 дней.

\section{ЛИТЕРАТУРА}

1. Baldwin RL. Rhinitis medicamentosa (an approach to treatment). J Med Assoc State Ala, 1975, 47: 33-35.

2. Fox N. Chronic effect of epinephrine and ephedrine on the nasal mucosa. Arch Otolaryngol, 1931, 30: 73-76.

3. Feinberg SM, Friedlaender S. Nasal congestion from frequent use of privine hydrochloride. $J$ Am Med Assoc, 1945, 128: 1095-1096. doi:10.1001/jama.1945.92860 320001011.

4. Lake CF. Rhinitis medicamentosa. Proc Staff Meet Mayo Clin, 1946, 21: 367-71.

5. Mortuairea G, de Gaboryb L, Francëois M, Massh̆d G, Bloche F, Brionf N, Jankowskig R, Serranoh E. Rebound congestion and rhinitis medicamentosa: Nasal decongestants in clinical practice. Critical review of the literature by a medical panel. European Annals of Otorhinolaryngology, Head and Neck diseases, 2013, 130: 137-144. doi: 10.1016/j.anorl.2012.09.005.

6. Graf PM, Hallen $\mathrm{H}$. Changes in nasal reactivity in patients with rhinitis medicamentosa after treatment with fluticasone propionate and placebo nasal spray. ORL J Otorhinolaryngol Relat Spe, 1998, 60: 334-338.

7. Graf P. Rhinitis medicamentosa: a review of causes and treatment. Treat Respir Med, 2005, 4 21-29.

8. Passali D, Salerni L, Passali GC, Passali FM, Bellussi L. Nasal decongestants in the treatment of chronic nasal obstruction: efficacy and safety of use. Expert Opin Drug Saf, 2006, 5: 783-790. doi: 10.1517/14740338.5.6.783.
9. Lockey RF. Rhinitis medicamentosa and the stuffy nose. J Allergy Clin Immunol, 2006, 118 1017-1018. doi:10.1016/j.jaci.2006.06.018.

10. Vaidyanathan $S$, Williamson $P$ Clearie $K$, Khan F, Lipworth B. Fluticasone reverses oxymetazolineinduced tachyphylaxis of response and rebound congestion. Am J Respir Crit Care Med, 2010, 182(1): 19-24. doi: 10.1164/rccm.200911-17010C

11. Graf P. Long-term use of oxy-and xylometazoline nasal sprays induces rebound swelling, tolerance, and nasal hyperreactivity. Rhinology,1996, 34: 9-13.

12. Graf P. Rhinitis medicamentosa: aspects of pathophysiology and treatment. Allergy, 1997, 52(40): 28-34.

13. Лопатин А.С. Сосудосуживающие препараты: механизмы действия, клиническое применение и побочные эффекты. Российская ринология, 2007, 1: 43-48.

14. Лопатин А.С. Ринит. Руководство для врачей М.: Лummeppa, 2010: 417.

15. Morris S, Eccles R, Martez SJ, Riker DK, Witek TJ, An evaluation of nasal response following different treatment regimens of oxymetazoline with references to rebound congestion. Am J Rhinol, 1997, 11: 109-115.

16. Yoo JK, Seikaly H, Calhoun KH. Extended use of topical nasal decongestants. Laryngoscope, 1997, 107: 40-43.

17. Watanabe H, Foo TH, Djazeri B. Oxymetazoline nasa spray three times daily for four weeks in normal subjects is not associated with rebound congestion or tachyphylaxis. Rhinology, 2003, 41: 167-174.

18. Lin CY, Cheng PH, Fang SY. Mucosal changes in rhinitis medicamentosa. Ann Otol Rhinol Laryngol, 2004, 113: 47-51.
19. Knipping S, Holzhausen HJ, Goetze G, Riedere A, Bloching MB. Rhinitis medicamentosa: electron microscopic changes of human nasal mucosa. Otolaryngol Head Neck Surg, 2007, 136: 57-61. doi:10.1016/j.otohns.2006.08.025.

20. Ramey JT, Bailen E, Lockey RF. Rhinitis medicamentosa. J Investig Allergol Clin Immunol, 2006, 16(3): 148-155.

21. Walker JS. Rhinitis medicamentosa. J Allergy, 1952, 23(2): 183-186.

22. Stephens AL, Boggs PB. Intranasal dexamethasone: an adjunct in the treatment of chemical rhinitis. Ann Allergy, 1968, 26: 612-613.

23. Hallen H, Enerdal J, Graf P. Fluticasone propionate nasal spray is more effective and has a faster onset of action than placebo in treatment of rhinitis medicamentosa. Clin. Ex Allergy, 1997, 27: 552-558.

24. Tas A, Yagiz R, Yalcin O, Uzun C, Huseyinova G, Adali MK, Karasalihoglu AR. Use of mometasone furoate aqueous nasal spray in the treatment of rhinitis medicamentosa: an experimental study. Otolaryngol Head Neck Surg, 2005, 132(4): 608-612. doi: 10.1016/j.otohns.2005.01.010.

25. Minshall E, Ghaffar O, Cameron L, O'Brien F, Quinn H, Rowe-Jones J, Davies RJ, Prior A, Lund VJ, Mackay IS, Nolop K, Lutsky B, Durham SR, Hamid Q. Assessment by nasal biopsy of long-term of mometasone furoate aqueous nasal spray (Nasonex) in the treatment of perennial rhinitis. Otolaryngol. Head Neck Surg, 1998, 118(5): 648-654.

26. Yuta A, Ogawa Y. Clinical review of 33 cases of rhinitis medicamentosa by decongestant nasal spray. Arerugi, 2013, 62(12): 1623-1630. 